\title{
(Re-)Organizing Health Systems
}

\section{Denise Felber Dietrich}

Secrétaire générale de Santé publique Suisse
Un système de santé doit poursuivre différents objectifs: respecter l'égalité des chances, être performant, garantir une offre en fonction des besoins tout en restant économiquement et financièrement fiable. En l'an 2000, l'Organisation mondiale de la santé (OMS) fixe des buts pour l'évaluation des systèmes de santé nationaux. Ceux-ci correspondent à la répartition de l'état de santé des populations, à la médecine en faveur du patient et à l'équité des charges financières [1] .

L'espérance de vie en Suisse a nettement augmenté depuis 1900, entre autre grâce à une offre sanitaire de qualité. Il est à préciser que le pourcentage des personnes de plus de 65 ans a pratiquement triplé. Chaque sixième personne est plus âgée que tion qui donnent matière à discuter. Un quart est porté par les ménages privés, 20\% par l'Etat et env. $40 \%$ par les assurances sociales [5]. Ceci signifie qu'en Suisse, contrairement aux pays limitrophes, une partie importante des coûts de la santé est prise en charge par les personnes privées, ceci indépendamment de leur situation financière. Les avis divergent quant à l'équité de cette répartition.

Les causes de l'explosion des coûts et les propositions pour freiner celle-ci font l'objet de discussions intenses. Les motifs en sont le développement démographique, une prévention insuffisante, le fédéralisme, une coordination déficiente, la puissance des groupes d'intérêts, les fausses incitations financières,

\section{«Les informations sur les inégalités en termes de santé sont plutôt}

\author{
rares en Suisse.»
}

65 ans [2]. La population vieillit non seulement, mais elle reste généralement en meilleure santé [3]. Parallèlement à l'augmentation de l'espérance de vie depuis des décennies, la mortalité des nourrissons a continuellement diminué. Aujourd'hui, en moyenne, seuls 4 de 1000 enfants nés vivant meurent [3]. La population elle-même est satisfaite de sa propre santé: $71 \%$ estime son état de santé comme très bon ou bon. Plus de trois quarts de la population est également satisfait du système de santé en Suisse [4]. Les informations sur les inégalités en termes de santé sont plutôt rares en Suisse et reflètent ainsi une mauvaise image de la répartition des facteurs de risque au sein de la population.

Bien qu'il reflète une image positive, le système de santé est un thème très présent dans les médias, lors de débats entre politiciens et notamment dans le milieu des spécialistes en santé publique. Ce sont principalement l'évolution des coûts et le financement des coûts de la santé en continuelle augmenta- une garantie de qualité insuffisante, des médicaments trop chers et l'évolution de la médecine technique. La responsabilité est en conséquence attribuée alternativement aux différents acteurs.

L'Organisation de coopération et de développement économique (OCDE) et l'OMS retiennent dans leur rapport paru l'année dernière que les décideurs politiques suisses devront à l'avenir relever des défis conséquents afin de pouvoir maintenir et améliorer les prestations du système de santé. Le développement démographique et les changements actuels dans le mode de vie laissent prévoir une augmentation des risques de santé au sein de la population vivant en Suisse. Les progrès médico-techniques s'ajoutant à cette tendance, il en résultera dans les décennies à venir, que davantage d'habitants et d'habitantes en Suisse souffriront d'une, voire de plusieurs maladies chroniques. Les exigences vis-àvis du système de santé suisse vont donc également augmenter [6].
Correspondance: Dr méd. et phil., MPH Denise Felber Dietrich Secrétaire générale Santé publique Suisse Effingerstrasse 54 CH-3001 Berne

denise.felber[at]public-health.ch

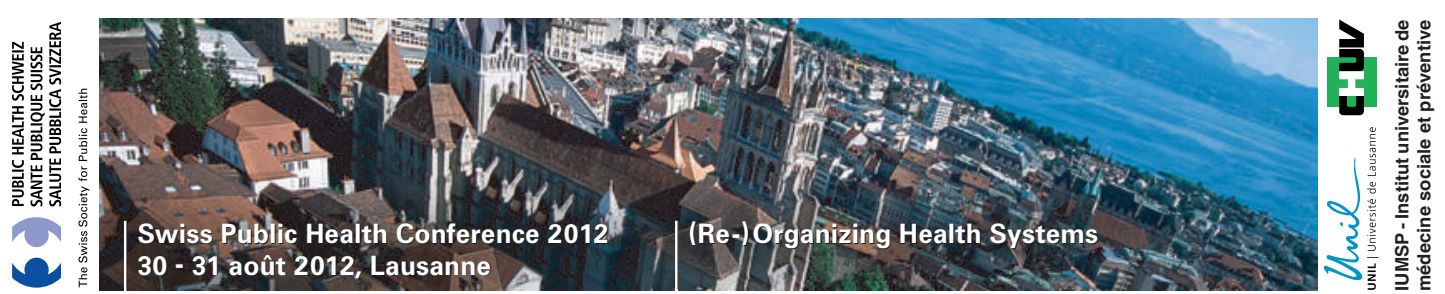


Dans son rapport, l'OCDE édicte maintes recommandations destinées à la Suisse et relatives aux diverses possibilités de gestion de ces défis telle que
Systems». Les différents acteurs de santé publique aborderont les thèmes susmentionnés. Venez participer aux différents débats à Lausanne!

\section{Le développement démographique et les changements actuels dans le mode de vie laissent prévoir une augmentation des risques de santé au sein de la population vivant en Suisse.}

l'amélioration de la gestion stratégique (Governance), ce qui implique ipso facto le renforcement et l'institutionnalisation de la coordination entre l'Etat et les cantons ainsi qu'entre les cantons eux-mêmes et l'amélioration du système d'information sur la santé. Le recensement de données comparables dans tout le pays sur les résultats sanitaires et la mortalité en relation avec les soins de santé est considéré comme indispensable pour le pilotage. Renforcer la planification stratégique et nationale pour le personnel de santé, relever l'importance de la prévention dans le système de santé suisse et appliquer les critères d'efficacité des coûts lors de la définition du catalogue des prestations sont des recommandations complémentaires citées par l'OCDE.

La Conférence Suisse de Santé Publique ouvre ses portes les 30 et 31 août prochains au CHUV à Lausanne sur le thème de «(Re-)Organizing Health

\section{Références}

1 Organisation mondiale de la santé (OMS). Rapport sur la santé dans le monde - Pour un système de santé plus performant. Genève; 2000.

2 Office fédéral de la statistique (OFS). Statistique de la population et des ménages (STATPOP). Neuchâtel; parutions diverses.

3 Office fédéral de la statistique (OFS). Annuaire statistique de la Suisse. Neuchâtel; parutions diverses.

4 Burla L. International Health Policy Survey 2010 des Commonwealth Fund. Analyse des résultats suisses à l'intention de l'Office fédéral de la santé publique (OFSP) (Obsan Dossier 15). Neuchâtel: Observatoire suisse de la santé; 2011.

5 Office fédéral de la statistique (OFS). Coût et financement du système de santé en 2010. Neuchâtel; 2012.

6 OCDE, Organisation mondiale de la santé (OMS). Examens de l'OCDE des systèmes de santé: Suisse 2011. Publication OCDE; 2011

\section{Swiss Public Health Conference 2012 à Lausanne, le 30 et 31 août}

Thèmes jeudi 30 août:

Governance of health systems

Preparing health care workforce
Thèmes vendredi 31 août:

Health systems research

Preventive strategies: roles, responsibilities, and partnership

Intervenants

Yann Bourgueil, Directeur Institut de recherche et documentation en économie de la santé, Paris Monika Diebold, Leiterin Schweizerisches Gesundheitsobservatorium, Neuchâtel

Catherine Gasser, Leiterin Abteilung Gesundheitsberufe, Bundesamt für Gesundheit, Bern

Niek Klazinga, OECD Health Division, Academic Medical Center University of Amsterdam

Pierre-François Leyvraz, Directeur général, Centre hospitalier universitaire vaudois, Lausanne

Pierre-Yves Maillard, Conseiller d'Etat, Dépt. de la santé et de l'action sociale, Lausanne

David McDaid, The London School of Economics and Political Science, London

George Mensah, Vice President Global Nutrition, PepsiCo, USA

Diane Morin, Directrice Institut universitaire de formation et de recherche en soins, Université de Lausanne

Nicole Probst-Hensch, Présidente de la Commission scientifique de Santé publique Suisse, Berne

Pascal Strupler, Directeur Office Fédérale de la Santé Publique, Berne

Walter Ricciardi, Director of the Department of Public Health, Università Cattolica del Sacro Cuore, Rome Ursula Zybach, Présidente Santé publique Suisse, Berne

La Conférence est organisée conjointement par Santé publique Suisse et l'Institut Universitaire de Médecine Sociale et Préventive de Lausanne.

D'autres informations et inscriptions sous www.conference.public-health.ch 